\title{
From D-galactose to enantiopure 3-azabicyclo[3.3.0]octen-7-one derivatives via Pauson-Khand reaction
}

\author{
Pilar Areces, ${ }^{* a}$ Esther Carrasco, ${ }^{a}$ and Joaquín Plumet ${ }^{* b}$ \\ ${ }^{a}$ Universidad de Extremadura. Facultad de Ciencias, Departamento de Química Orgánica, \\ 06071 Badajoz, Spain \\ ${ }^{b}$ Universidad Complutense. Facultad de Química, Departamento de Química Orgánica, \\ 28040 Madrid, Spain \\ E-mail:pareces@unex.es; plumety@quim.ucm.es
}

Dedicated to Professor José Elguero on his $70^{\text {th }}$ birthday

(received 02 Dec 04; accepted 08 Mar 05; published on the web 13 Mar 05)

\begin{abstract}
The 8-substituted-3-azabicyclo[3.3.0]octen-2-one-7-derivatives $\mathbf{1 8}$ and $\mathbf{1 9}$ have been obtained in enantiomerically pure form from D-galactose in acceptable chemical yield (30\%, nine steps) and moderate diastereomeric ratio $(\mathbf{1 8 : 1 9}=2.5: 1)$ via intramolecular Pauson-Khand $(\mathrm{PK})$ reaction of the appropriate enyne.
\end{abstract}

Keywords: Intramolecular Pauson-Khand reaction, cyclopentenones, carbohydrates

\section{Introduction}

The cobalt-mediated carbonylative co-cyclization of an alkene and an alkyne, the PausonKhand, (PK), reaction is a flexible method for the synthesis of cyclopentenones. ${ }^{1}$ The enantioselective version of this reaction using a chiral inductor covalently bonded to the alkene or the alkyne moiety had led in some cases to useful results. ${ }^{2}$ By the use of carbohydrates as sources of chirality, these compounds have been considered as chiral substrates in the intramolecular PK reaction. ${ }^{3}$ However, the introduction as chiral auxiliaries in these reactions has not been reported previously. Our group has recently reported the first case, ${ }^{4}$ using compound $\mathbf{1}$ derived from D-glucal, obtaining approximately 1:1 mixtures of the diastereomers 2 and 3 (Scheme 1). In order to improve the diastereoselectivity of this reaction we heeded a report by Mulzer et al. ${ }^{5}$ who observed that compounds 4 and 5, when treated with dicobalt-octacarbonyl with subsequent thermal decomposition, gave mixtures of diastereomers 6 and 7 in 3:1 and 1:1 ratios, respectively. Thus, the diastereoselectivity of these reactions depends upon the relative stereochemistry of the benzoyl protecting group in $\mathbf{4}$ and $\mathbf{5}$ (Scheme 2). 


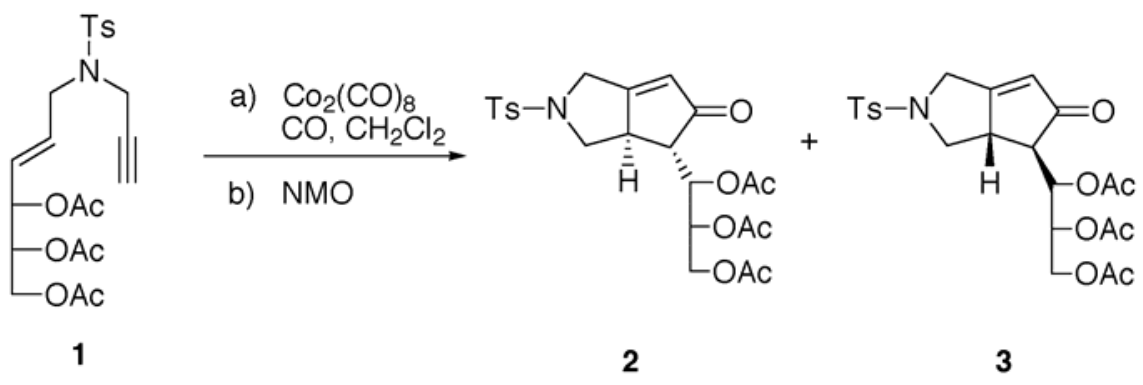

Scheme 1. Intramolecular PK reaction of (2E)-4,5,6-tri-O-acetyl-1,2,3-trideoxy-1-( $N$ propargyltosylamine)-D-erythro-hex-2-enose (1).

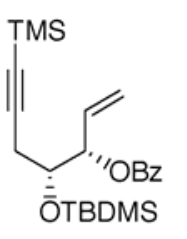

4

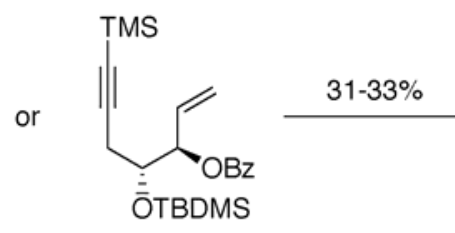

5

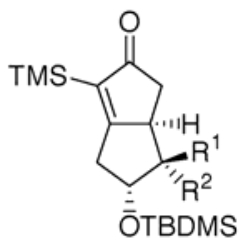

6a, $R^{2}=\mathrm{OBz}, \mathrm{R}^{1}=\mathrm{H}$

7a, $R^{1}=O B z, R^{2}=H$

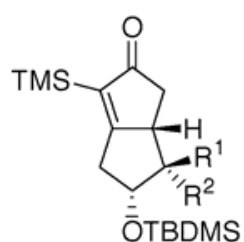

6b, $R^{2}=O B z, R^{1}=H \quad 6 a: 6 b=3: 1$ 7b. $R^{1}=O B z, R^{2}=H \quad 7 a: 7 b=1: 1$

Scheme 2. PK cyclizations of chiral substrates 4 and 5.

With this precedent in mind we speculated that the inversion of the stereochemistry of the stereogenic center at the allylic position of the sugar chain could improve the diastereomeric ratio for the resulting cyclopentenones. We now report our results in this field.

\section{Results and Discussion}

The aza-enyne 8 having the D-galacto configuration at the sugar chain was used as starting material (Scheme 3). This compound was obtained from tetra-O-acetyl-D-galactal 11 through a synthetic sequence involving the ring opening to give the aldehyde 13, formation of the propargyl imine 14, reduction, and protection of the resulting amine with tosyl chloride. On the other hand, tetra-O-acetyl-D-galactal $\mathbf{1 1}$ was synthesized from D-galactose $\mathbf{9}$ in two steps without isolation of the intermediate bromide $10 .{ }^{6-8}$ 

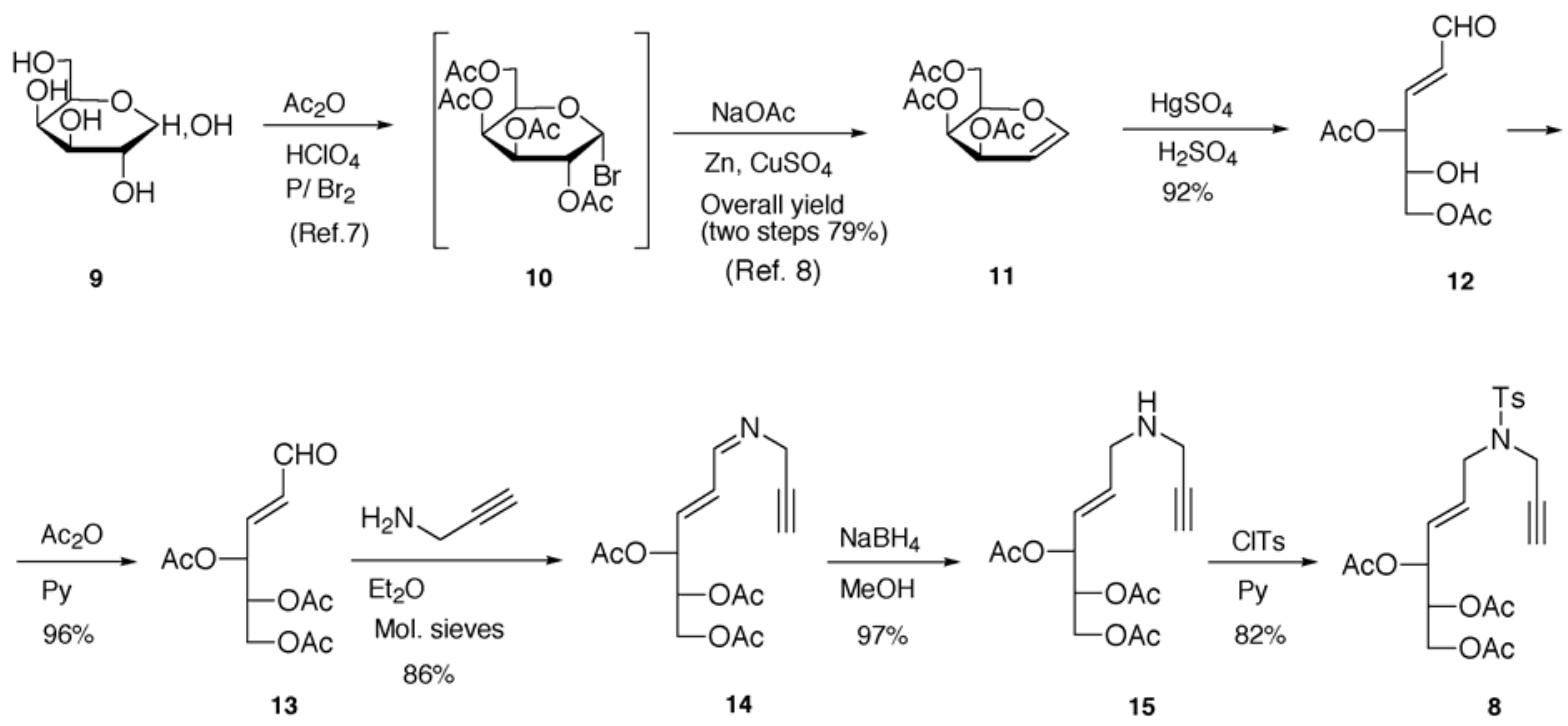

Scheme 3. Synthesis of aza-enyne 8 from D-galactose.

Having compound 8 in hand, its reaction with $\mathrm{Co}_{2}(\mathrm{CO})_{8}$ during $15 \mathrm{~min}$ in $\mathrm{CH}_{2} \mathrm{Cl}_{2}$ at $\mathrm{RT}$ (18$20^{\circ} \mathrm{C}$ ) under $\mathrm{CO}$ atmosphere led to complete formation of the complex 16 (Scheme 4, $\mathrm{R}_{\mathrm{F}} 0.39$ hexane-ethyl acetate $2: 1$ ) which by reaction with $\mathrm{N}$-methylmorpholine $\mathrm{N}$-oxide (NMO) for $1 \mathrm{~h}$ at $\mathrm{RT}$ in $\mathrm{CH}_{2} \mathrm{Cl}_{2}$ afforded a mixture of compound 17 (5\%) and cyclopentenones 18 and 19 (61\% isolated overall yield) in a ratio 18:19 $=2.5: 1$. From this crude reaction mixture, compound 17 $\left(\mathrm{R}_{\mathrm{F}}\right.$ 0.76, hexane-ethyl acetate 1:2) was isolated. On the other hand, fractional crystallization (hexane-ethyl acetate, 1:1) of the mixture 18:19 allowed the isolation of pure 18 [mp $147^{\circ} \mathrm{C}$, $\left.[\alpha]_{\mathrm{D}}-123^{\circ}\left(\mathrm{c} 0.5, \mathrm{CHCl}_{3}\right)\right]$.

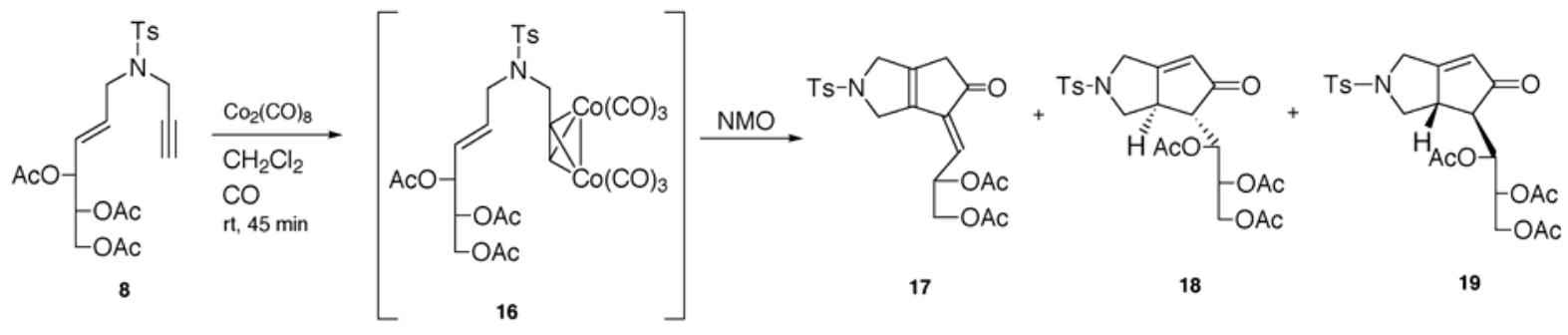

Scheme 4. Intramolecular Pauson-Khand reaction of the chiral enyne 8.

The structural determination of compounds 18 and 19 was achieved on the following basis. The X-ray analysis of compound $3^{4}$ shows that, in the solid state, the conformation of this compound is that in which the tosyl group is located under the bicyclic ring (Figure 1). The ${ }^{1} \mathrm{H}-$ NMR spectrum also indicates that the solution-conformations of $\mathbf{3}$ and $\mathbf{2}$ might be similar. Thus, the upfield-shifted peaks for hydrogens $\mathrm{H}-3 a$ and $\mathrm{H}-4$ show that both protons lie in the shielding region of the aryl group (Figure 1). ${ }^{9}$ Under these conditions the signal for hydrogen $\mathrm{H}-3 a$ is 
shifted to low-field in the diastereomer having the sugar chain in the $\alpha$-position compared to the diastereomer with the sugar chain in the $\beta$-position. Similarly, the H-4 signal is shifted upfield in the diastereomer with the sugar chain in the $\alpha$-position. Assuming that the same conformational trend should be expected for compounds 18 and 19 we deduced that the major compound 18 must have an $\alpha$-sited chain whereas compound 19 must show the opposite configuration.

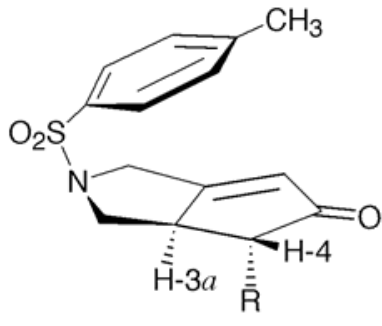

2, 18

\begin{tabular}{l|l}
$\begin{array}{l}\text { Chain Conf. = D-erithro } \\
\text { (Compounds 2 and 3) }\end{array}$ & $\delta \mathrm{H}_{3 a}: 3.34 \mathrm{ppm}$ \\
& $\delta \mathrm{H}_{4}: 2.39 \mathrm{ppm}$ \\
& $\delta \mathrm{H}_{3 a}: 3.26 \mathrm{ppm}$ \\
$\begin{array}{l}\text { Chain Conf. = D-threo } \\
\text { (Compounds 18 and 19) }\end{array}$ & $\delta \mathrm{H}_{4}: 2.39 \mathrm{ppm}$
\end{tabular}

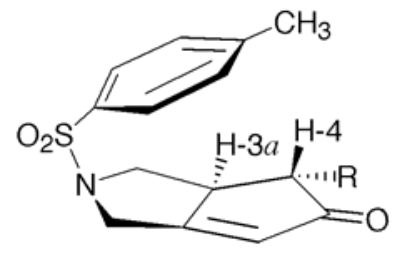

3, 19

$\begin{array}{lll}\delta \mathrm{H}_{3 a}: & 3.09 \mathrm{ppm} & 0.25 \\ \delta \mathrm{H}_{4}: & 2.47 \mathrm{ppm} & -0.08 \\ & & \\ \delta \mathrm{H}_{3 a}: & 2.61 \mathrm{ppm} & 0.65 \\ \delta \mathrm{H}_{4}: & 2.52 \mathrm{ppm} & -0.13\end{array}$

Figure 1. Conformations and chemical shifts for compounds 2, 3, 18 and 19.

Compound 17 arises from the formal hydrogenolytic cleavage of Pauson-Khand adducts with concomitant double bond migration. This type of products from Pauson-Khand adducts are often obtained and, in some cases, this reaction has been synthetically useful. ${ }^{10}$ It should be noted that compound 17 was also obtained by reaction of compound 3 with pyridine at $60{ }^{\circ} \mathrm{C}(27 \%$ isolated yield). ${ }^{4}$ On this basis we propose the reaction path given in Scheme 5 for the formation of compound 17. 


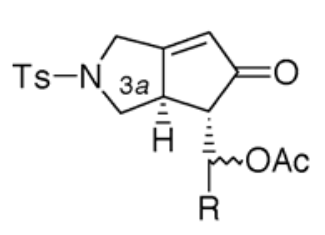

18 and/or 19
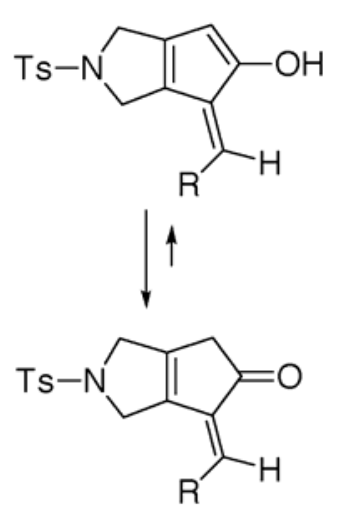

17

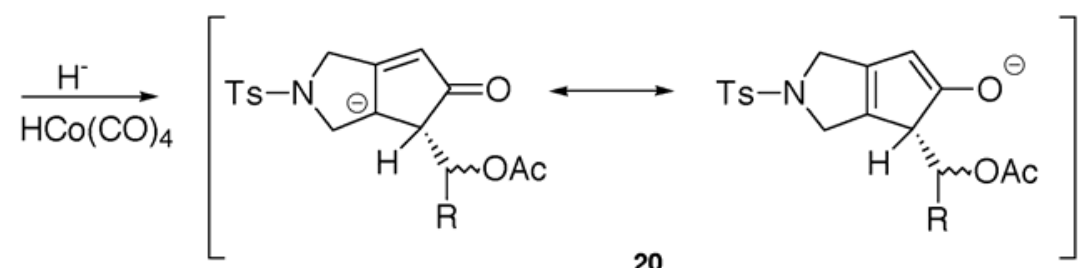

Solv-H
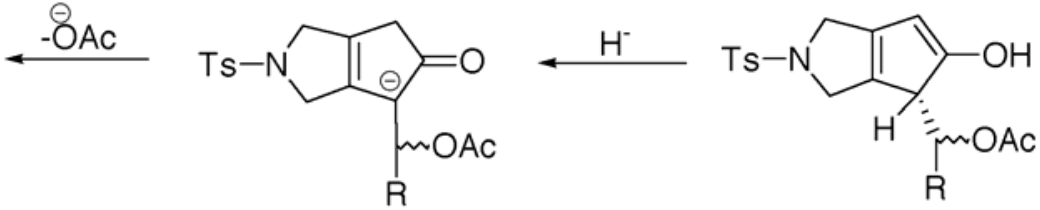

21

Scheme 5. Reaction path for the formation of $\mathbf{1 7 .}$

In this case, a hydride ion generated from the species $\mathrm{HCo}(\mathrm{CO})_{4}{ }^{11}$ abstracts the acidic proton $\mathrm{H}-3 a$ from the resulting cyclopentanone, yielding the extended enolate ion 20. Protonation (probably by traces of water present in the NMO) affords 21, which can again generate a conjugate carbanion after abstraction of proton H-4. Finally, the protonation during the work-up yields compound 17.

Considering the generally accepted mechanism for the intramolecular PK cycloaddition ${ }^{12}$ made explicit for our cases, the observed diastereomeric ratio should depend on the steric demand in the insertion step of the alkene, thereby yielding the corresponding couple of diastereomers (Scheme 6).

Two approaches are possible for the alkene insertion step (Scheme 7) giving the two diastereomeric bicyclopentenones. In the approach (a) there are no important steric interactions between the sugar chain and the cobalt-alkyne complex moiety whereas in (b) the steric interference between the remote $\mathrm{Co}(\mathrm{CO})_{3}$ moiety and $\mathrm{R}^{\prime}$ appears to be evident. Considering the distance between $\mathrm{R}^{\prime}$ and the $\mathrm{Co}(\mathrm{CO})_{3}$ group, this steric interaction induces only a relatively small diastereomeric ratio (2.5:1). In the case of the related compounds 2 and $\mathbf{3}$ derived from Dglucal this interaction occurs between a sterically less demanding acetoxy group an the remote $\mathrm{Co}(\mathrm{CO})_{3}$ moiety, and no diastereoselectivity was observed at all. ${ }^{4}$ In summary, in this report we have shown that the inversion of the configuration at the allylic position of the sugar chain produces a significant change of the stereochemical outcome in the intramolecular PK reaction. 
Although a tentative model has been proposed, additional work is necessary to clarify the precise origin of this change in diastereoselectivity.
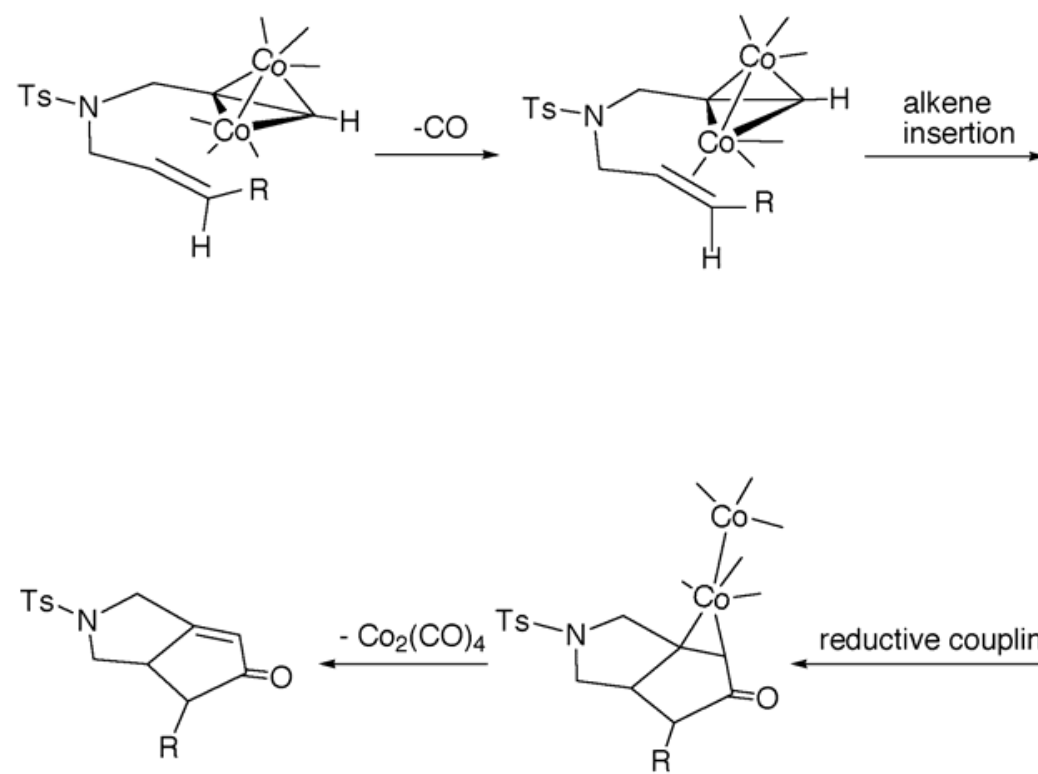

$\stackrel{\mathrm{Co}_{2}(\mathrm{CO})_{4}}{\longleftarrow}$

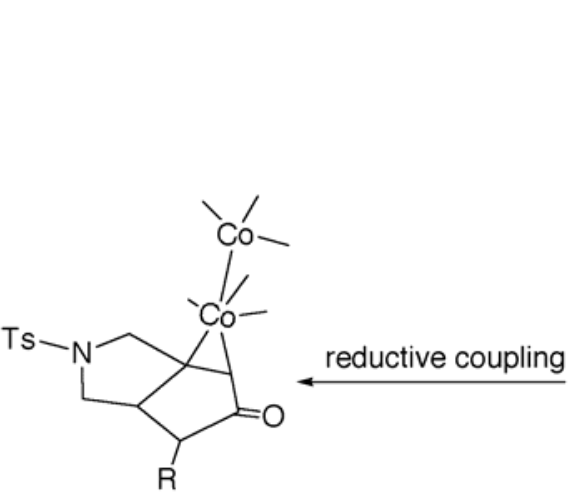

Scheme 6. Mechanism proposed for the Pauson-Khand cycloaddition (CO ligands are omitted).

a)
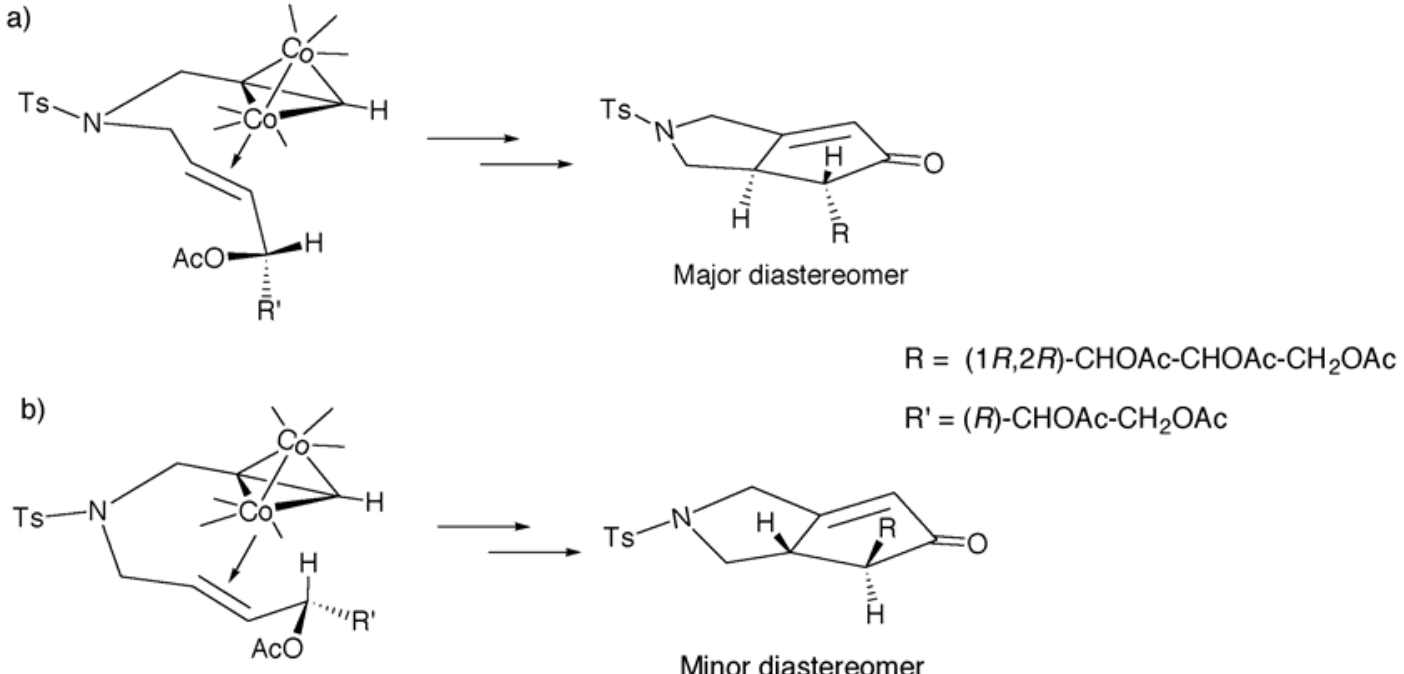

Scheme 7. Formation of major and minor diastereomers from different conformers of the dicobalt-hexacarbonyl-enyne complexes. 


\section{Experimental Section}

General Procedures. IPK reactions were carried out under argon atmosphere. Anhydrous $\mathrm{CH}_{2} \mathrm{Cl}_{2}$ was distilled from sodium hydride immediately prior to use. Melting points were determined with a capillary apparatus and are uncorrected. Optical rotations were measured at 25 $\pm 2^{\circ} \mathrm{C}$ with a Perkin-Elmer 241 polarimeter. Analytical and preparative TLC were performed on Merck $60 \mathrm{GF}_{254}$ silica gel with monitoring by UV light at 254 and $360 \mathrm{~nm}$ or by exposure to iodine vapor. Flash chromatography ${ }^{1}$ was performed on Merck 60 silica gel (230-400 mesh). Specific rotations $[\alpha]_{\mathrm{D}}{ }^{20}$ were determined using a Perkin Elmer Polarimeter 141. IR spectra were recorded on a Perkin-Elmer 399 or a Midac FT-IR spectrometer. Solid samples were run as $\mathrm{KBr}$ disks and liquids as thin films on $\mathrm{NaCl}$ plates. Details are reported as $v_{\max } / \mathrm{cm}^{-1} .{ }^{1} \mathrm{H}$ - and ${ }^{13} \mathrm{C}-$ NMR spectra were obtained on a Bruker AM 400 instrument at 400 and $100 \mathrm{MHz}$, respectively, or on a Bruker $\mathrm{AC} 200$ instrument at 200 and $500 \mathrm{MHz}$, respectively, in $\mathrm{CDCl}_{3}\left(\mathrm{Me}_{4} \mathrm{Si}\right.$ as internal standard) unless otherwise specified. Elemental analyses were carried out using a Leco 932 analyzer at the Universidad de Extremadura. Mass spectra $\left(\mathrm{HRMS} / \mathrm{CI}^{+}\right)$were recorded on a VG Autospec spectrometer; only significant fragment ions are reported.

(2E)-4,6-Di-O-acetyl-2,3-dideoxy-aldehydo-D-threo-hex-2-enose (12). Mercuric sulfate $(0.30 \mathrm{~g}, 0.57 \mathrm{mmol})$ was added to a stirred solution of $\mathbf{1 1}(6.20 \mathrm{~g}, 26.9 \mathrm{mmol})$ in 1,4-dioxane $(32 \mathrm{ml})$ and $5 \mathrm{mM}$ sulfuric acid $(120 \mathrm{ml})$, and stirring was continued for $3 \mathrm{~h}$; an excess of barium carbonate was added $(0.39 \mathrm{~g}, 2.03 \mathrm{mmol})$, the suspension was shaken and filtered, and the filtrate was evaporated, giving a clear oil (5.69 g, 92\%); IR (film) $3460 \mathrm{~m}, 1741 \mathrm{~s}, 1690 \mathrm{~s}, 1231 \mathrm{~s} \mathrm{~cm}^{-1}$. ${ }^{1} \mathrm{H}-\mathrm{NMR}(400 \mathrm{MHz}) \delta 9.60$ (d, J $\mathrm{J}_{1,2} 7.6 \mathrm{~Hz}, \mathrm{H}-1$ ), 6.84 (dd, J J,4 $4.4 \mathrm{~Hz}, \mathrm{~J}_{3,2} 15.6 \mathrm{~Hz}, \mathrm{H}-3$ ), 6.29 (ddd, $\mathrm{J}_{2,4} 1.3 \mathrm{~Hz}, \mathrm{H}-2$ ), 5.64 (m, H-4), 4.22 (dd, J = 3.6 Hz, J = 10.4 Hz, H-5), 4.11 (m, H-6, 2H), 2.18 (s, 3H, OAc), 2.11 (s, 3H, OAc); ${ }^{13} \mathrm{C}-\mathrm{NMR}(100 \mathrm{MHz}) \delta 192.3$ (C-1), 170.8, $169.3(2 \mathrm{O}-$ $\mathrm{CO}-\mathrm{CH}_{3}$ ), 149.2 (C-3), 132.8 (C-2), 72.0 (C-4), 70.1 (C-5), 64.3 (C-6), 20.4 (2 O-CO-CH$)_{3}$.

(2E)-4,5,6-Tri-O-acetyl-2,3-dideoxy-aldehydo-D-threo-hex-2-enose (13). A solution of the diacetate $12(5.82 \mathrm{~g}, 21.4 \mathrm{mmol})$ in acetic anhydride $(25 \mathrm{ml})$ and pyridine $(25 \mathrm{ml})$ was kept for 4 $\mathrm{h}$, poured onto a slurry of ice and dilute $\mathrm{HCl}(280 \mathrm{ml})$, and extracted three times with $\mathrm{CHCl}_{3}$; the extracts were combined, successively washed with aqueous $\mathrm{NaHCO}_{3}$ solution and water, dried, and evaporated to an oil (5.63 g, 97\%); [ $\alpha]_{\mathrm{D}}+59.2\left(\right.$ ( $\left.0.5 \mathrm{CHCl}_{3}\right)$; IR (film) $1750 \mathrm{~s}, 1690 \mathrm{~m}, 1220$ $\mathrm{s} \mathrm{cm}^{-1} .{ }^{1} \mathrm{H}-\mathrm{NMR}(400 \mathrm{MHz}) \delta 9.59$ (d, $\mathrm{J}_{1,2} 7.6 \mathrm{~Hz}, \mathrm{H}-1$ ), 6.24 (ddd, $\mathrm{J}_{2,3} 15.8, \mathrm{~J}_{2,4} 1.5 \mathrm{~Hz}, \mathrm{H}-2$ ), 6.73 (dd, $\left.\mathrm{J}_{3,4} 4.6 \mathrm{~Hz}, \mathrm{H}-3\right), 5.77$ (m, J $\left.\mathrm{J}_{4,5} 6.1 \mathrm{~Hz}, \mathrm{H}-4\right), 5.35$ (m, J $\mathrm{J}_{5,6} 5.6 \mathrm{~Hz}, \mathrm{H}-5$ ), 4.35 (dd, J $\mathrm{J}_{6,6}$ $12.0 \mathrm{~Hz}, \mathrm{H}-6), 4.00$ (dd, H-6'), 2.17 (s, 3H, OAc), 2.09 (s, 3H, OAc), 2.06 (s, 3H, OAc); ${ }^{13} \mathrm{C}-$ NMR (100 MHz) $\delta 192.2(\mathrm{C}-1), 170.3,169.7,169.3\left(3 \mathrm{O}-\mathrm{CO}-\mathrm{CH}_{3}\right), 133.2(\mathrm{C}-2), 148.5(\mathrm{C}-3)$, 70.2, 70.1 (C-4, C-5), 61.6 (C-6), 20.6, 20.5, (3 O-CO-CH ${ }_{3}$; HRMS Calcd for $\mathrm{C}_{12} \mathrm{H}_{16} \mathrm{O}_{7}+\mathrm{H}$ : 273.0974; Found 273.0983; m/z (rel. int.) 213 (M+H-AcOH, 14), 153 (M+H-2AcOH, 21). (2E)-Tri-O-acetyl-2,3-dideoxy-aldehydo-D-threo-hex-2-enose propargylimine (14). To a stirred solution of $13(3.52 \mathrm{~g}, 12.86 \mathrm{mmol})$ in dry ethyl ether $(25 \mathrm{~mL})$ at $0{ }^{\circ} \mathrm{C}$ was added propargylamine $(0.81 \mathrm{~mL}, 11.9 \mathrm{mmol})$ and molecular sieve (Merck $4 \AA$ ). After stirring for $1.5 \mathrm{~h}$ 
at RT, filtration and concentration yielded 14 as an oil $(3.01 \mathrm{~g}, 86 \%) ;[\alpha]_{\mathrm{D}}+24.4\left(c 0.5, \mathrm{CHCl}_{3}\right)$; IR (film) $3280 \mathrm{~m}, 1755 \mathrm{~s}, 1660 \mathrm{~m}, 1220 \mathrm{~s} \mathrm{~cm}^{-1} ;{ }^{1} \mathrm{H}-\mathrm{NMR}(400 \mathrm{MHz}) \delta 8.12$ (dd, J $\mathrm{J}_{1,2} 8.8 \mathrm{~Hz}, \mathrm{~J}_{1,1}$ ' 1.6 Hz, H-1), 6.40 (ddd, $\mathrm{J}_{2,3} 15.8 \mathrm{~Hz}, \mathrm{~J}_{2,4} 1.1 \mathrm{~Hz}, \mathrm{H}-2$ ), 6.18 (dd, J $\mathrm{J}_{3,4} 5.5 \mathrm{~Hz}, \mathrm{H}-3$ ), 5.66 (ddd, J J,5 $5.3 \mathrm{~Hz}, \mathrm{H}-4), 5.30$ (m, H-5), 4.34 (dd, $\mathrm{J}_{6,6}, 12.0 \mathrm{~Hz}, \mathrm{~J}_{5,6} 4.1 \mathrm{~Hz}, \mathrm{H}-6$ ), 4.07 (dd, $\mathrm{J}_{5,6}, 6.5 \mathrm{~Hz}, \mathrm{H}-6^{\prime}$ ), 4.46 (t, 2H, H-1'), 2.45 (t, $\left.\mathrm{J}_{1^{\prime} 3^{\prime}} 2.3 \mathrm{~Hz}, \mathrm{H}-3^{\prime}\right), 2.13$ (s, 3H, OAc), 2.09 (s, 3H, OAc), 2.06 (s, 3H, OAc); ${ }^{13} \mathrm{C}-\mathrm{NMR}(100 \mathrm{MHz}){ }^{\mathrm{TM}}$ 170.4, 169.7, 169.2 (3 O-CO-CH3), 161.9 (C-1), 136.7 (C-3), 132.5 (C-2), 78.2 (C-3'), 75.6 (C-2'), 70.6, 70.5 (C-4, C-5), 61.7 (C-6), 46.7 (C-1'), 20.5, 20.4 (3 O-CO-CH ${ }_{3}$ ); HRMS Calcd for $\mathrm{C}_{15} \mathrm{H}_{19} \mathrm{NO}_{6}+\mathrm{H}$ : 310.1290; Found 310.1307; m/z (rel. int.) 310 (M+H, 11), 273 (59), 212 (41), 170 (100), 153 (99).

(2E)-4,5,6-Tri-O-acetyl-1,2,3-trideoxy-1-propargylamine-D-threo-hex-2-enose (15). To a stirred solution of imine $14(5.9 \mathrm{~g}, 19.07 \mathrm{mmol})$ in $\mathrm{MeOH}(32 \mathrm{~mL})$ at $0^{\circ} \mathrm{C}$, sodium borohydride ( $0.72 \mathrm{~g}, 19.07 \mathrm{mmol})$ was added. The mixture was stirred for $15 \mathrm{~min}$ at RT, filtered, and the filtrate diluted with $\mathrm{CH}_{2} \mathrm{Cl}_{2}(130 \mathrm{~mL})$, then washed successively with sat. aq. $\mathrm{NaHCO}_{3}(2 \mathrm{x}$ $100 \mathrm{~mL})$ and water $(2 \times 100 \mathrm{~mL})$, dried with $\mathrm{MgSO}_{4}$ and concentrated yielding 15 as an oil $(5.7 \mathrm{~g}, 97 \%) ;[\alpha]_{\mathrm{D}}+9.0$ (c 0.5, $\mathrm{CHCl}_{3}$ ); IR (film) $3280 \mathrm{~m}, 1745 \mathrm{~s}, 1660 \mathrm{~d}, 1225 \mathrm{~s} \mathrm{~cm}^{-1}$; ${ }^{1} \mathrm{H}-\mathrm{NMR}$ $\left(400 \mathrm{MHz}_{3}\right)^{\text {тм }} 5.87$ (dt, $\left.\mathrm{J}_{1,2} 5.8 \mathrm{~Hz}, \mathrm{~J}_{2,3} 15.2 \mathrm{~Hz}, \mathrm{H}-2\right), 5.60$ (ddt, J $\mathrm{J}_{3,4} 6.4 \mathrm{~Hz}, \mathrm{~J}_{1,3} 1.2 \mathrm{~Hz}, \mathrm{H}-3$ ), 5.47 (t, $\left.\mathrm{J}_{4,5} 6.5 \mathrm{~Hz}, \mathrm{H}-4\right), 5.20$ (m, H-5), 4.31 (dd, J $\left.6_{6,6}, 12.1 \mathrm{~Hz}, \mathrm{H}-6\right), 4.05$ (dd, H-6'), 3.38 (d, J $1^{\prime}, 3^{\prime}$ 2.4 Hz, H-1'a, H-1'b), 3.31 (dd, H-1a, H-1b), 2.22 (t, H-3'), 2.08 (s, 3H, OAc), 2.06 (s, 3H, OAc), 2.05 (s, 3H, OAc); ${ }^{13} \mathrm{C}-\mathrm{NMR}(100 \mathrm{MHz}){ }^{\text {TM }} 170.4,170.0,169.6\left(3 \mathrm{O}-\mathrm{CO}-\mathrm{CH}_{3}\right), 133.8(\mathrm{C}-2)$, 125.3 (C-3), 81.6 (C-3'), 71.8 (C-2'), 71.6 (C-5), 71.2 (C-4), 62.1 (C-6), 49.1 (C-1), 37.2 (C-1'), 20.8, 20.7, 20.6 (3 O-CO-CH $)$; HRMS Calcd for $\mathrm{C}_{15} \mathrm{H}_{21} \mathrm{NO}_{6}+\mathrm{H}: 312.1447$; Found 312.1463; $\mathrm{m} / \mathrm{z}$ (rel. int.) 312 ( $\mathrm{M}+\mathrm{H}, 100), 252$ (M+H-AcOH, 49), 192 (M+H-2AcOH, 37).

(2E)-4,5,6-Tri- $O$-acetyl-1,2,3-trideoxy-1-( $N$-propargyltosylamino)-D-threo-hex-2-enose (8). A stirred solution of $\mathbf{1 5}(2.29 \mathrm{~g}, 7.37 \mathrm{mmol})$ in pyridine $(14 \mathrm{~mL})$ at $0{ }^{\circ} \mathrm{C}$ was treated with tosyl chloride $(4.20 \mathrm{~g}, 22.1 \mathrm{mmol})$. The mixture was kept $48 \mathrm{~h}$ at $4{ }^{\circ} \mathrm{C}$ and then poured into water/ice $(140 \mathrm{~mL})$, and extracted with $\mathrm{Et}_{2} \mathrm{O}(3 \times 70 \mathrm{~mL})$. The combined organic layers were rinsed successively with $2 \mathrm{~N}$ aq. $\mathrm{HCl}(3 \times 70 \mathrm{~mL})$, a saturated solution of $\mathrm{NaHCO}_{3}(3 \times 70 \mathrm{~mL})$ and brine $(3 \times 70 \mathrm{~mL})$, dried over $\mathrm{MgSO}_{4}$ and filtered. Evaporation of the solvent (reduced pressure) afforded $\mathbf{8}$ as an oil which was purified by flash chromatography (hexane-ethyl acetate 2:1) $(1.87 \mathrm{~g}, 82 \%) ;[\alpha]_{\mathrm{D}}+8.6\left(\mathrm{c} 0.5, \mathrm{CHCl}_{3}\right)$; IR (film) $3280 \mathrm{~m}, 1745 \mathrm{~s}, 1210 \mathrm{~s}, 1160 \mathrm{~s} \mathrm{~cm}^{-1} ;{ }^{1} \mathrm{H}-$ NMR (400 MHz) TM 7.72 (d, H-2", H-6"), 7.30 (d, H-3", H-5"), 5.70 (dt, J Ja, 5.6 Hz, J,3 $15.2 \mathrm{~Hz}$, H-2), 5.65 (dd, J $\mathrm{J}_{3,4} 5.8 \mathrm{~Hz}, \mathrm{H}-3$ ), 5.46 (t, $\mathrm{J}_{4,5} 5.6 \mathrm{~Hz}, \mathrm{H}-4$ ), 5.21 (ddd, $\mathrm{J}_{5,6} 4.1 \mathrm{~Hz}, \mathrm{~J}_{5,6^{\prime}} 6.4 \mathrm{~Hz}, \mathrm{H}-$ 5), 4.30 (dd, $\left.\mathrm{J}_{6,6^{\prime}} 11.9, \mathrm{~Hz}, \mathrm{H}-6\right), 4.04$ (bt, $\mathrm{J}_{1^{\prime} \mathrm{a}, 3^{\prime}}=\mathrm{J}_{1^{\prime} \mathrm{b}, 3^{\prime}} 2.5 \mathrm{~Hz}, \mathrm{H}-\mathrm{l}^{\prime} \mathrm{a}, \mathrm{H}-\mathrm{l}^{\prime} \mathrm{b}$ ), 4.01 (dd, H-6'), 3.82 (d, $\left.\mathrm{J}_{1 \mathrm{~b}, 2} 4.0 \mathrm{~Hz}, 2 \mathrm{H}-1\right), 2.43$ (s, 3H, $\mathrm{CH}_{3}-\mathrm{Ar}$ ), 2.09 (s, 3H, OAc), 2.08 (s, 3H, OAc), 2.05 (s, 3H, OAc), 2.02 (t, H-3'); ${ }^{13} \mathrm{C}-\mathrm{NMR}(100 \mathrm{MHz})$ тм 170.4, 169.9, 169.5 (3 O-CO-CH$), 143.6$ (C-3", C-5"), 135.6 (C-1"), 129.5 (C-4"), 129.1 (C-2), 128.9 (C-3), 127.6 (C-2", C-6"), 76.2 (C-2'), 73.9 (C-3'), 71.2 (C-4), 71.0 (C-5), 61.9 (C-6), 47.5 (C-1), 35.9 (C-1'), $21.4\left(\mathrm{CH}_{3}-\mathrm{Ar}\right), 20.8,20.7,20.6$ (3 O-CO- $\mathrm{CH}_{3}$ ); HRMS Calcd for $\mathrm{C}_{22} \mathrm{H}_{27} \mathrm{NO}_{8} \mathrm{~S}-\mathrm{OAc}$ : 406.1324; Found 406.1324; m/z (rel. int.) 
406 (M-AcOH, 63), 346 (M-2AcOH, 13), 310 (M-Ts, 47), 286 (M-3AcOH, 9), 250 (M-Ts$\mathrm{AcOH}, 23)$.

4-(2',3'-Diacetoxypropyliden)-2-tosyl-2,3,4,6-tetrahydro-1H-cyclopenta-[c]-pyrrole-5-one (17), (3aR,4S)-4-(tri-O-acetyl-D-threo-triol-1-yl)-2-tosyl-2,3,3a,4-tetrahydro-1H-cyclopenta[c]-pyrrole-5-one (18) and (3aS,4R)-4-(tri-O-acetyl-D-threo-triol-1-yl)-2-tosyl-2,3,3a,4tetrahydro-1H-cyclopenta-[c]-pyrrole-5-one (19). A solution of aza-enyne 8 (449 mg, $0.96 \mathrm{mmol})$ in freshly distilled $\mathrm{CH}_{2} \mathrm{Cl}_{2}(5.5 \mathrm{ml})$ was treated with $\mathrm{Co}_{2}(\mathrm{CO})_{8}(405 \mathrm{mg}, 1.05 \mathrm{mmol})$ under $\mathrm{CO}$ atmosphere and the mixture was stirred for $45 \mathrm{~min}$. Then NMO (665 $\mathrm{mg}, 5.78 \mathrm{mmol})$ was added portionwise over a 15 -min period, cooling the reaction at $0{ }^{\circ} \mathrm{C}$ before each addition and allowing it to reach RT before the next one. The mixture was stirred for $1 \mathrm{~h}$ at RT, diluted with $\mathrm{CH}_{2} \mathrm{Cl}_{2}(50 \mathrm{ml})$, washed successively with $2 \mathrm{M} \mathrm{HCl}(2 \times 50 \mathrm{ml})$, sat. $\mathrm{NaHCO}_{3}(2 \times 50 \mathrm{ml})$, and water $(2 \times 50 \mathrm{ml})$, dried $\left(\mathrm{MgSO}_{4}\right)$ and concentrated in vacuo. The reaction mixture was eluted through a short column (1 cm long) of $\mathrm{SiO}_{2}$ (hexane/ethyl acetate $3: 1 \rightarrow$ ethyl acetate) to afford an initial fraction $\left(\mathrm{R}_{\mathrm{F}}\right.$ 0.80) (hexane-ethyl acetate 1:2) of the intermediate dicobalthexacarbonyl complex $16(103 \mathrm{mg})$, a second fraction $\left(\mathrm{R}_{\mathrm{F}} 0.76\right)$ of the cyclopentenone 17 $(18.8 \mathrm{mg}, 4 \%)$ and a third fraction of a mixture $\left(\mathrm{R}_{\mathrm{F}} 0.46\right)$ of 18 and $19(2.5: 1,247 \mathrm{mg}, 61 \%)$ which was resolved by fractional crystallization (hexane/ethyl acetate $1: 1$ ).

18: $\mathrm{mp} 147^{\circ} \mathrm{C} ;[\alpha]_{\mathrm{D}}-123\left(\mathrm{c} 0.5 \mathrm{CHCl}_{3}\right)$; IR (KBr) $1735 \mathrm{~s}, 1700 \mathrm{~s}, 1640 \mathrm{~m}, 1600 \mathrm{w}, 1340 \mathrm{~s}, 1220$ s, $1155 \mathrm{~s} \mathrm{~cm}^{-1}$; 'H-NMR (400 MHz) тм 7.74 (d, H-2", H-6"), 7.36 (d, H-3", H-5"), 5.99 (bs, H-6), $5.60\left(\mathrm{t}, \mathrm{J}_{1^{\prime}, 4}=\mathrm{J}_{1^{\prime}, 2^{\prime}} 4.2 \mathrm{~Hz}, \mathrm{H}-1^{\prime}\right), 5.36$ (m, H-2'), 4.36 (bd, J $\left.{ }_{1 \mathrm{a}, 1 \mathrm{~b}} 16.9 \mathrm{~Hz}, \mathrm{H}-1 \mathrm{~b}\right), 4.26$ (dd, J $2^{\prime}, 3^{\prime} \mathrm{b}$ $\left.4.8 \mathrm{~Hz}, \mathrm{~J}_{3^{\prime} \mathrm{a}, 3^{\prime} \mathrm{b}} 11.8 \mathrm{~Hz}, \mathrm{H}-3^{\prime} \mathrm{b}\right), 4.01$ (bd, J $\left.\mathrm{la}, 1 \mathrm{~b} 16.9 \mathrm{~Hz}, \mathrm{H}-1 \mathrm{a}\right), 3.99$ (m, 2H, H-3b, H-3'a), 3.26 (m, $\mathrm{H}-3 a), 2.67$ (dd, $\mathrm{J}_{3 \mathrm{a}, 3 a}=\mathrm{J}_{3 \mathrm{a}, 3 \mathrm{~b}} 10.2 \mathrm{~Hz}, \mathrm{H}-3 \mathrm{a}$ ), 2.45 (s, 3H, $\mathrm{CH}_{3}-\mathrm{Ar}$ ), 2.39 (t, J ${\text { 1', } 4^{\prime}}^{\prime}=\mathrm{J}_{3 a, 4} 3.2 \mathrm{~Hz}, \mathrm{H}-$ 4), 2.11 (s, 3H, OAc), 2.06 (s, 3H, OAc), 2.02 (s, 3H, OAc); ${ }^{13} \mathrm{C}-\mathrm{NMR}(100 \mathrm{MHz})$ тм 203.9 (C5), 177.5 (C-6a), 170.4, 169.8, 169.1 (3 O-CO-CH $), 144.3$ (C-1"), 133.2 (C-4"), 130.0 (C-3", C5"), 127.4 (C-2", C-6"), 124.9 (C-6), 71.1 (C-2'), 68.2 (C-1'), 61.8 (C-3'), 53.0 (C-4), 52.0 (C-3), 47.5 (C-1), 45.9 (C-3a), $21.5\left(\mathrm{CH}_{3}-\mathrm{Ar}\right), 20.8,20.6,20.4$ (3 O-CO-CH$)_{3}$. Anal. Calcd for $\mathrm{C}_{23} \mathrm{H}_{27} \mathrm{NO}_{9} \mathrm{~S} ; \mathrm{C}, 55.97 ; \mathrm{H}, 5.51 ; \mathrm{N}, 2.83 ; \mathrm{S}, 6.49$. Found C, 55.76; H, 5.54; N, 2.95; S, 6.30. HRMS calcd for $\mathrm{C}_{23} \mathrm{H}_{27} \mathrm{NO}_{9} \mathrm{~S}+\mathrm{H}$ : 494.1484, found: 494.1477; m/z (rel. int.) $434(\mathrm{M}+\mathrm{H}-\mathrm{AcOH}$, 23), $374(\mathrm{M}+\mathrm{H}-2 \mathrm{AcOH}, 71), 314(\mathrm{M}+\mathrm{H}-3 \mathrm{AcOH}, 42), 218(\mathrm{M}-2 \mathrm{AcOH}-\mathrm{Ts}, 11), 158$ (M$3 \mathrm{AcOH}-\mathrm{Ts}, 17)$.

\section{Acknowledgements}

This work was supported by MCYT-Spain, BQU2003-04967-C02-01 and the Regional Government of Extremadura (Spain), 2PR02A016. E.C. acknowledges a fellowship from the MECD-Spain. 


\section{References and Notes}

1. For selected recent reviews, see: (a) Sugihara, M.; Yamaguchi, M.; Nishizawa, M. Chem. Eur. J. 2001, 7, 1589. (b) Hanson, B.E. Comments on Inorg. Chem. 2002, 23, 289. (c) Rivero, M.R.; Adrio, J.; Carretero, J.C. Eur. J. Org. Chem. 2002, 2881. (d) Pericás, M.A.; Basells, J.; Castro, J.; Marchueta, J.; Moyano, A.; Riera, A.; Vázquez, J.; Verdaguer, X. Pure Appl. Chem. 2002, 74, 167. (e) Gibson, S.E.; Stevanazzi, A. Angew. Chem., Int. Ed. 2003, 42, 1800. (f) Blanco-Urgoiti, J.; Anorbe, L.; Pérez-Serrano, L.; Domínguez, G.; PérezCastells, J. Chem. Soc. Rev. 2004, 33, 32. (g) Boñaga, L.V.R.; Krafft, M.E. Tetrahedron 2004, 60, 9795.

2. For a concise and complete review, see: Ingate, S.T.; Marco-Contelles, J. Org. Prep. Proc. Int. 1998, 30, 121.

3. See ref. 2, p.133, and Marco-Contelles, J.; de Opazo, E. J. Carbohydr. Chem. 2002, 21, 201 and references therein.

4. Areces, P.; Durán, M.A.; Plumet, J.; Hursthouse, M.B.; Light, M.E. J. Org. Chem. 2002, 67, 3506.

5. Mulzer, J.; Graske, K.D.; Kirste, B. Ann. 1988, 891.

6. Although compound $\mathbf{1 1}$ is commercially available (Aldrich, $\mathbf{5} \mathrm{g}, 118.8 €$ ), this product can be obtained on the multi-gram scale $(21.4 \mathrm{~g})$ according to the described procedure at the price of $5 \mathrm{~g} \sim 0.50 €$.

7. (a) Levene, P.A.; Tipson, R.S. J. Biol. Chem. 1931, 93, 631. (b) Helferich, E.N.; Mulcahy, E.N.; Ziegler, H. Ber. 1954, 87, 233.

8. González, F.; Lesage, S.; Perlin, A. Carbohydr. Res. 1975, 42, 267.

9. Similar observations have been previously reported in related compounds. See: Yoo. S.; Lee, S. J. Org. Chem. 1999, 59, 6968.

10. (a) Kerr, W.J.; McLaughlin, M.; Pauson, P.L.; Robertson, S.M. Chem. Commun. 1999, 2171. (b) Arjona, O.; Csaky, A.G.; Murcia, C.; Plumet, J. J. Org. Chem. 1999, 64, 7338.

11. Several processes of isomerization of cyclopentenones obtained in PK reactions have been described (See ref. 1g). Experimental evidence indicates that those reactions were catalyzed by the hydride $\mathrm{HCo}(\mathrm{CO})_{4}$ generated by reduction of $\mathrm{Co}_{2}(\mathrm{CO})_{8}$ in the reaction media. See, for example, Davies, S.G. In Organotransition Metal Chemistry: Applications to Organic Synthesis, Pergamon Press: Oxford, 1982; p 367.

12. See refs. $1 \mathrm{f}$ and $1 \mathrm{~g}$ and references cited therein. 